\section{Kompass \\ Autoimmun}

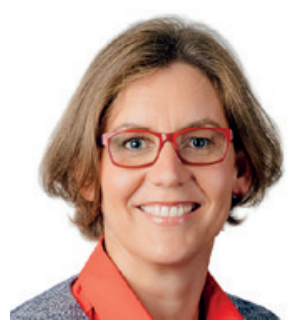

Irina Blumenstein

Medizinische Klinik 1, Universitätsklinikum Frankfurt, Frankfurt am Main, Deutschland

\title{
Gestörte intestinale Barriere triggert das Immunsystem
}

Sehr geehrte Leserinnen und Leser!

Kennen Sie das? Sie erhalten in Ihrer Post unzählige medizinische Zeitschriften, Sie überfliegen die Überschriften und werfen sie dann nach kurzem Überlegen in den Altpapiermüll.

Warum könnte es sich lohnen, dieses Heft nicht nur zu überfliegen, sondern es auf den Stapel «unbedingt lesen» zu legen? In dieser Ausgabe des Kompass Autoimmun geht es um einige faszinierende Aspekte rund um chronisch-entzündliche Darmerkrankungen (CED). Diese umfassen die Colitis ulcerosa und den Morbus Crohn und sind heute präziser als «autoinflammatorische» Erkrankungen zu bezeichnen - deren exakte Ursache wir im Übrigen immer noch nicht kennen! Wir gehen davon aus, dass eine gestörte intestinale Barriere eine pathologische Aktivierung des Immunsystems in Gang setzt und vor allem aufrechterhält. Wir behandeln diese überschießende Immunantwort mehr oder minder spezifisch mit den uns zur Verfügung stehenden immunsuppressiven Therapien.

Eine sehr spannende Erweiterung unseres Therapieportfolios könnte die Ernährungs- therapie sein. Viele Patienten fragen nach einer Anleitung zur Änderung ihrer Ernährungsgewohnheiten. Sie bemerken, dass ihre gastrointestinalen Beschwerden in Abhängigkeit von ihrer Ernährungsweise stark variieren. Diese Beobachtung geht dabei über das Weglassen von Milchprodukten und das Vermeiden von Weizen hinaus. Von einer möglicherweise ergänzenden diätetischen Therapie der CED sind wir selbstverständlich noch weit entfernt, zumal diese ja bekanntermaßen auch sehr schwer dauerhaft umzusetzen ist. Attraktiv bleibt das Konzept jedoch in jedem Fall, gibt es den Patienten doch die Möglichkeit einer Beteiligung an der Therapie, von den sehr günstigen Kosten und den äußerst geringen $\mathrm{Ne}$ benwirkungen ganz zu Schweigen. Das Team um Luc Biedermann bringt Sie in einem sehr lesenswerten Artikel auf den neuesten Stand der Erkenntnisse rund um die Ernährungsintervention bei CED.

Glücklicherweise sind wir als Gastroenterologen in der Betreuung von CED-Patienten nicht allein: Wir stehen tagtäglich in Austausch mit Chirurgen, Dermatologen, Rheumatologen, Radiologen, Nephrologen, Psychologen, Physiotherapeuten und Sozial- arbeitern. Alle diese Kontakte müssen jedoch im Einzelfall mühsam hergestellt werden und nicht immer gelingt eine zeitnahe Vorstellung bei den Kollegen.

Ein Beispiel eines gelebten interdisziplinären Austausches finden Sie in diesem Heft in Form eines Interviews mit Kollegen aus der Gastroenterologie und Chirurgie zum Thema chirurgische Interventionen bei CED.

Die zahlreiche Etablierung von sogenannten Entzündungszentren, in welchen eine enge Zusammenarbeit der im wesentlichen beteiligten Disziplinen in räumlicher Nähe möglich ist, ist sicherlich ein Schritt in die richtige Richtung einer umfassenden und verbesserten Versorgung von Patienten mit CED.

In diesem Sinne wünsche ich Ihnen eine gesunde Zeit und ein wenig Ruhe zum Abschalten und Lesen.

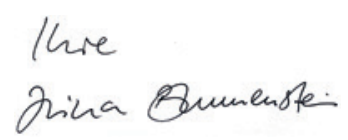

PD Dr. Irina Blumenstein information@karger.com @ 2020 S. Karger GmbH, Freiburg www.karger.com/kai

\section{Karger"'}

